\title{
Biomechanics in orthodontics - A review (Part 1)
}

\author{
Khalid Ashraf $^{1}$, Rohit Kulshrestha ${ }^{2, *}$, Ragni Tandom³ ${ }^{3}$ Harmeet Kaur ${ }^{4}$, Pranshu Mathur ${ }^{5}$ \\ ${ }^{\mathbf{1} O r t h o d o n t i s t, ~ S P E S ~ H o s p i t a l, ~ T o h a n a ~ H a r y a n a, ~}{ }^{2}$ Senior Lecturer, Dept. of Orthodontics \& Dentofacial Orthopedics, Terna \\ Dental College, Navi Mumbai, Maharashtra, ${ }^{3}$ Professor and Head, ${ }^{5}$ Post Graduate Student, Dept. of Orthodontics \& Dentofacial \\ Orthopedics, Saraswati Dental College, Lucknow, Uttar Pradesh, ${ }^{4}$ Post Graduate Student, Dept. of Orthodontics \& Dentofacial \\ Orthopedics, BBD Dental College, Lucknow, Uttar Pradesh, India
}

*Corresponding Author: Rohit Kulshrestha

Email: kulrohit@gmail.com

\begin{abstract}
Orthodontic forces can be dealt with numerically as vectors. At the point when in excess of one power is connected to a tooth, the forces can be consolidated to decide a solitary by and large resultant. Forces can likewise be isolated into segments so as to decide impacts parallel and perpendicular to the occlusal plane, Frankfort horizontal, or the long axis of the tooth. Forces produce either translation (bodily movement), rotation, or a combination of translation and rotation, depending upon the relationship of the line of action of the force to the center of resistance of the tooth. Since most forces are applied at the bracket, it is important to process equal force frameworks at the centre of resistance keeping in mind the end goal to anticipate tooth development. The point of this review article is to give us a knowledge on biomechanics in orthodontics which are utilized as a part of day by day practice.
\end{abstract}

Keywords: Force, Moment, Couple, Biomechanics.

\section{Introduction}

"All you can do is to push or pull or turn a tooth. I have given you an appliance. Now for God's sake, use it!" (Edward $\mathrm{H}$ Angle). ${ }^{1}$ Indeed Angle gave us a number of appliances, and so did other pioneers in orthodontics. At the outset, we should emphasize what Weinstein has said so succinctly, "there is only one disease - malocclusion. The medicine is force, and the number of ways to apply that force! The biologic cascade of events that ultimately results in bone remodeling and orthodontic tooth movement begins with the mechanical activation of an orthodontic appliance. ${ }^{2}$ The force systems produced by orthodontic appliances, consisting of both forces and moments, displace teeth in a manner that is both predictable and controllable. By varying the ratio of moment to force applied to teeth, the type of tooth movement experienced can be regulated by the orthodontist.

Orthodontic appliances obey the laws of physics and can be activated to generate the desired force systems to achieve predetermined treatment goals for individual patients. Likewise, any orthodontic appliance can be analyzed to define the mechanical force systems it produces. Understanding the biomechanical principles underlying orthodontic appliance activations is essential for executing efficient and successful orthodontic treatment. The forces produced by orthodontic appliance originate primarily in elastically deformed wires. These wires absorb and release energy during loading and unloading. Therefore, the behaviour of elastic materials should be considered in the design of orthodontic appliance system through which mechano-therapy is delivered. If the pressure is of the proper intensity and duration and if there are no restraining environmental or functional forces, the tooth, or teeth, will move. ${ }^{3}$ Most of the early appliances were of the removable type. Actually they are modified dentures. The early fixed appliances were usually crude metal bands ligated to the teeth with brass or silver wire. They were outstanding for their inefficiency and their ability to trap food. Kinglsy, Angle, Case and others soon realized that for any effective tooth movement some means must be devised to control the individual teeth. This led to the development of attachments that were soldered on modified crowns or bands. ${ }^{4}$

The earliest attachments were nothing more than spurs that would hold a wire. The average appliance consisted of two molar bands "anchor" bands, or modified crowns, with long tubes or sheaths on the buccal surface, parallel with the occlusal plane, and a heavy labial arch wire that followed the contour of the upper and lower dental arch. Individual teeth that were irregular were banded with copper, brass and silver strip material, and spurs were soldered to permit the rotation and tipping of these teeth. ${ }^{5}$ Rotation was accomplished by tying or ligating the individual teeth to the arch wire. All movements were accomplished by tipping the teeth out toward the arch wire. Pioneer orthodontists who worked with the ligature wires and crude bands with spurs soon learned that simple tipping of the teeth did not provide the desired tooth movement. It became apparent that to achieve the proper tooth position and axial inclination of the teeth, one must have better control of each individual tooth -- control that will permit the movement of the root of the tooth as well as the crown. The purpose of this article was to review and discuss the principles of biomechanics associated with different tooth movements and to summarise its effect on the dentition. ${ }^{6}$ 


\section{Definitions in Biomechanics ${ }^{7}$}

Mass: Mass applies to every particle of matter in the universe. The mass of any body is the quantity of matter it contains. An upper permanent first molar, for example, has a greater mass than a lower central incisor.

Center of Mass: Many bodies behave as if their mass were concentrated at a single point. If for example you were out in space one day enjoying a nice space walk and happened across a large box that you wanted to push out of the way, you could push this box away from you in several different ways. You could push it at one of its edges and it would start tumbling away from you, or you could push it in line with its center of mass and it would leave you without tumbling end over end. The box would be translating away from you i.e; every point on the box would be travelling in a parallel, straight line. The box would behave as if its entire mass were concentrated at that single point, its center of mass.

Force: A load connected to a question that will tend to move it to an alternate position in space. Force however inflexibly characterized in units of Newton's (mass times the acceleration of gravity), is generally estimated in weight units of grams or ounces.

Centre of Resistance: A point at which resistance to movement can be concentrated for scientific investigation. For an object in free space, the centre of resistance is the same as the centre of mass. In the event that the object is halfway controlled, just like the case for a fence post reaching out into the earth or a tooth root inserted in bone, the centre of resistance will be dictated by the idea of the outer requirements. The centre of resistance for a tooth is at the rough midpoint of the embedded bit of the root (i.e., about somewhere between the root summit and the peak of the alveolar bone).

Moment: A measure of the inclination to pivot a protest around some point. A moment is produced by a power acting at a separation. Quantitatively, it is the result of the force times the perpendicular distance from the point of force application to the force of resistance and therefore is estimated in units of gram-millimeter (or proportional). In the event that the line of activity of a connected force does not go through the center of resistance, a moment is fundamentally made. Not exclusively will the force have a tendency to move the object, moving it to an alternate position, it additionally will have a tendency to turn the object around the center of resistance. This, obviously, is accurately the circumstance when a force is connected to the crown of a tooth. Not exclusively is the tooth moved toward the force, it additionally turns around the center of resistance - subsequently the tooth tips as it moves.

Couple: Two forces equal in magnitude and opposite in direction. The aftereffect of applying two forces along these lines is an unadulterated moment, since the translatory impact of the forces offsets. A couple will create unadulterated rotation, turning the object around its center of resistance, while the combination of a force and a couple can change the manner in which a question pivots while it is being moved.

Center of Rotation: The point around which rotation really happens when an object is being moved. At the point when two forces are applied in combination to an object, the center of rotation can be controlled and made to have any coveted area. The use of a force and a couple to the crown of a tooth, truth be told, is the system by which bodily movement of a tooth, or considerably more noteworthy movement of the root than the crown, can be created.

\section{Basics of Biomechanics ${ }^{8}$}

Center of resistance is defined by its relationship to the force: a force for which the line of activity goes through the center of resistance creates a development of unadulterated translation. For a tooth this movement can be mesiodistal or vestibulolingual, intrusive or extrusive. Everyone or free body has one point on which it can (from a certain perspective) be flawlessly adjusted. This point is known as the center of gravity. For some physical figurings, it seems as though whatever is left of the object does not exist, and the majority of the weight is amassed at this single point. The movement of a free body relies on the relationship of the line of activity of the force to the center of gravity. Teeth are not allowed to move in light of a force; rather, they are controlled by periodontal structures, which are not uniform around the root of a tooth along these lines, a direct practically equivalent to toward the centre of gravity is utilized; this is known as the center of resistance. By definition, a force with a line of activity going through the center of resistance produces translation. (Fig. 1)

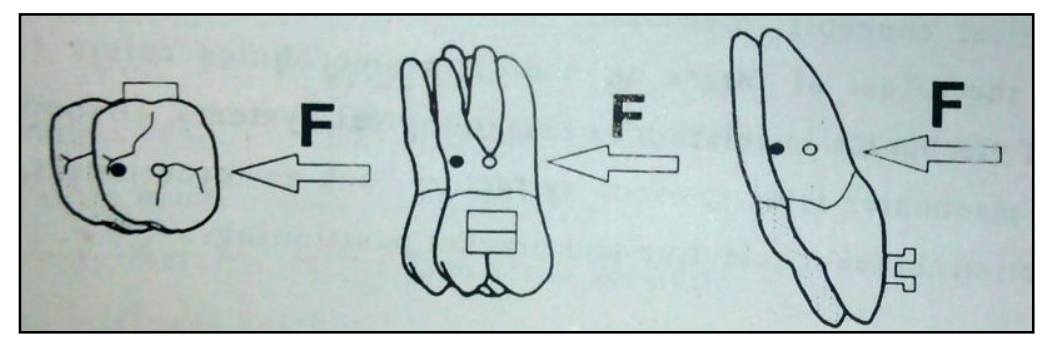

Fig. 1: Force acting through the center of resistance will make all points on the tooth move a similar amount in that similar direction. This is named translation and is conceivable toward any path 
The exact location of the center of resistance is not easily identified. The center of resistance of a tooth is dependent on:

The correct area of the center of resistance isn't effectively recognized. The center of resistance of a tooth is subject to:

1. The quantity of roots

2. The root length and morphology

3. The level of the alveolar bone.

\section{Number of Roots}

The center of resistance of a solitary rooted tooth is on the long axis of the tooth, most likely between $33 \%$ and one half of the root length apical to the alveolar crest. $^{2}$ For a multirooted tooth, the center of resistance is presumably between the roots, maybe a couple millimeters apical to the furcation ${ }^{9-10}$ (Fig. 2)
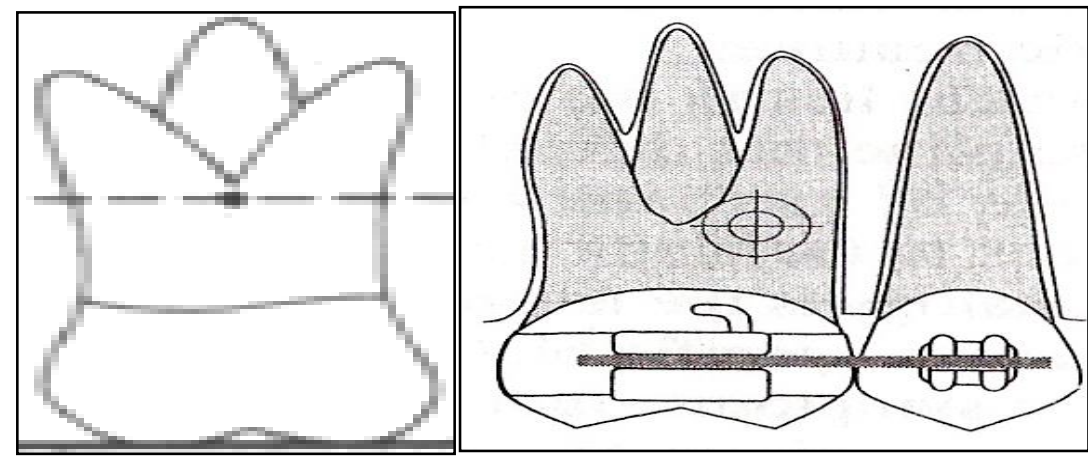

Fig. 2: Number of the roots

\section{Length and Morphology of Roots}

Maxillary canines have longer roots than lateral incisors hence they will have a center of resistance farther from the bracket (Fig. 3). Since the tooth movement coming about because of a force conveyed at the bracket relies on the distance of the line of action of the force from the center of resistance, identical forces applied to teeth with various root lengths can have diverse impacts.

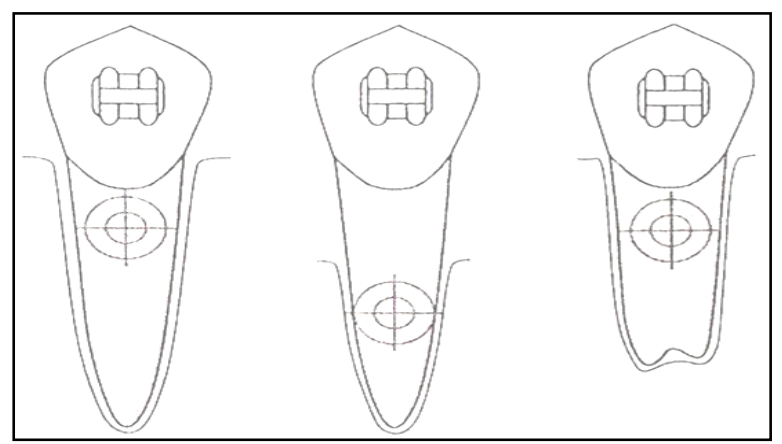

Fig. 3: Length and morphology of root

Level of the Alveolar Bone: The movement of the teeth in grown-ups with alveolar bone loss will be unique in relation to in youths ${ }^{2}$ (Fig. 4).

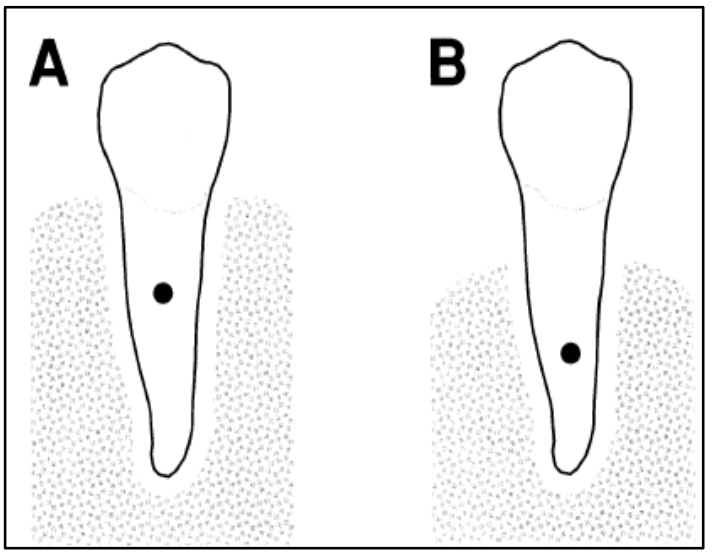

Fig. 4: The center of resistance in a tooth with full periodontal support (A). The center of resistance is more apical in a periodontally traded off tooth $(B)$.

\section{Force}

Force is defined as a follow up on the body that progressions or tends to change the condition of rest or of uniform movement of the body. Forces are vectors, having both direction and magnitude. ${ }^{8}$ Changing the direction, size or point of force application will influence the nature of the tooth displacement that will happen. The extent of the vector speaks to its size and its line of activity, sense and a point of origin depicts direction. Force application will influence the nature of tooth relocation that will happen. Expanding the extent of the force will build the measure of tooth relocation at first. Be that as it may, it is vague how force is identified with the rate of tooth movement, which is organically controlled phenomenon. ${ }^{11}$

The purpose of force application additionally has an impact on the nature of tooth movement. Forces, as vectors, can be joined or isolated scientifically. At least 
two forces following up on a solitary point can be included utilizing straightforward trigonometry or vector expansion and spoke to as a solitary force by then. ${ }^{8}$ For instance, isolate istally and extrusively coordinated forces can be consolidated into one distalextrusive power (Fig. 5). Thus a solitary distal extrusive force can be settled numerically into its distal and extrusive segments.

\section{Types of forces in orthodontics ${ }^{12}$ :}

1. Continuous - force kept up at some apparent part of the first starting with one patient visit then onto the next (Fig. 6B).

2. Interrupted-drive levels decay to zero between 3.

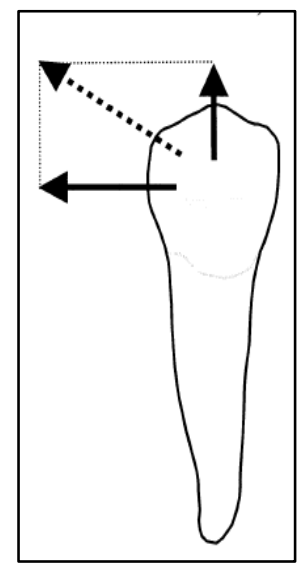

Fig. 5: Forces are vectors and can be combined or resolved mathematical

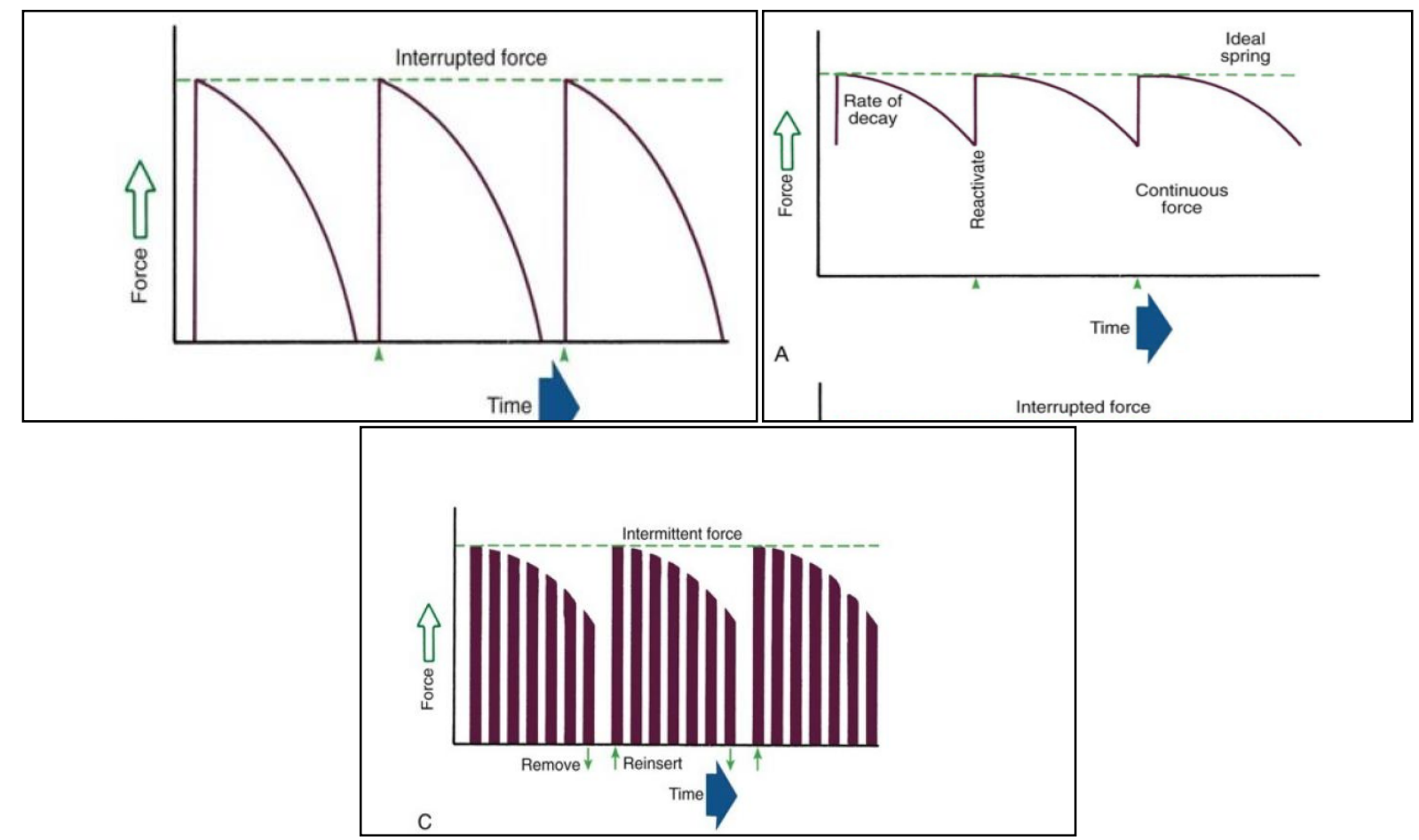

Fig. 6: Continuous force (B), Interrupted force (A), Intermittent force (C)

Both continuous and interrupted forces can be produced by fixed appliances that are constantly present.

Intermittent: Force levels decline abruptly to zero intermittently, when an orthodontic appliance or elastic attached to a fixed appliance is removed by the patient, and then return to the original level some time later (Fig. 6C). When tooth movement occurs, force levels will decrease as they would with a fixed appliance (i.e., the intermittent force can also become interrupted between adjustments of the appliance). 
Intermittent forces are produced by all patientactivated appliances such as removable plates, headgear, and elastics. Forces generated during normal function (e.g., chewing, swallowing, speaking) can be viewed as a special case of intermittently applied forces, most of which are not maintained for enough hours per day to have significant effects on the position of the teeth.

\section{Moment of Force ${ }^{13}$}

As per Lindauer SJ, a moment is characterized as the inclination to turn and may allude to rotation, tipping or torque in orthodontic wording. At the point when a force is applied other than through the center of resistance, notwithstanding moving the center of resistance toward constrain, a moment is made. In the event that a distal force is connected buccal to the center of resistance, the center of resistance of the tooth will move distally and the tooth will pivot mesiobucally (Fig. 7A). In the event that the distal force is applied coronal to the center of resistance, as when an elastic chain is extended over the bracket on a premolar, the center of resistance will move distally and the crown of the tooth will tip distally (Fig.7B). In the event that the meddlesome force is connected to the center of resistance, the center of resistance will interrupt and the crown of the tooth will torque facially (Fig. 7C).

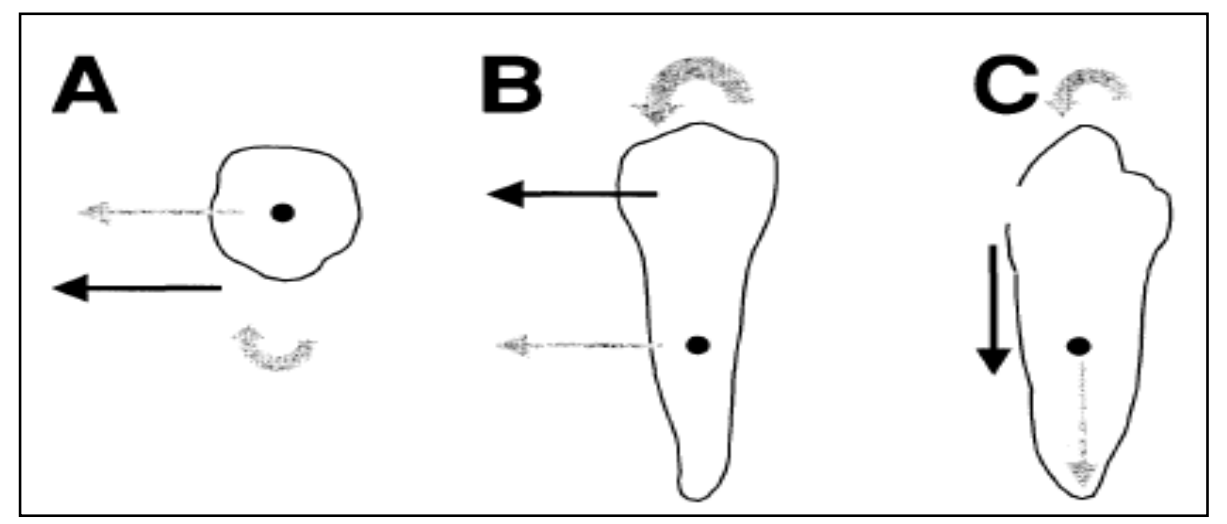

Fig. 7: A power connected at the bracket (dark) will bring about both a force and moment at the center of resistance (gray). Occlusal view; (A): buccal view; (B), mesial view; (C). Applied forces and couples are appeared in black all through. Proportionate forces frameworks (forces and moments) at the center of resistance are appeared in gray.

A force connected straightforwardly through the center of resistance will cause unadulterated translation of the tooth toward the force with no rotation. ${ }^{14}$ In the event that a similar force is applied away from the center of resistance, towards the crown for instance, the tooth will move toward the force and there will be a moment made to tip the crown toward the force (Fig. 8A). Expanding the extent of the force (Fig. 8B), or applying a similar force much further from the center of resistance will build the inclination for pivot. In this manner the extent of a moment $(\mathrm{M})$ is equivalent to the size of the applied force (F) times the distance (d) of that force from the center of resistance, $\mathrm{M}=\mathrm{Fd}$. The distance is constantly estimated perpendicular from the line of activity of the force to the center of resistance (Fig. 5C).

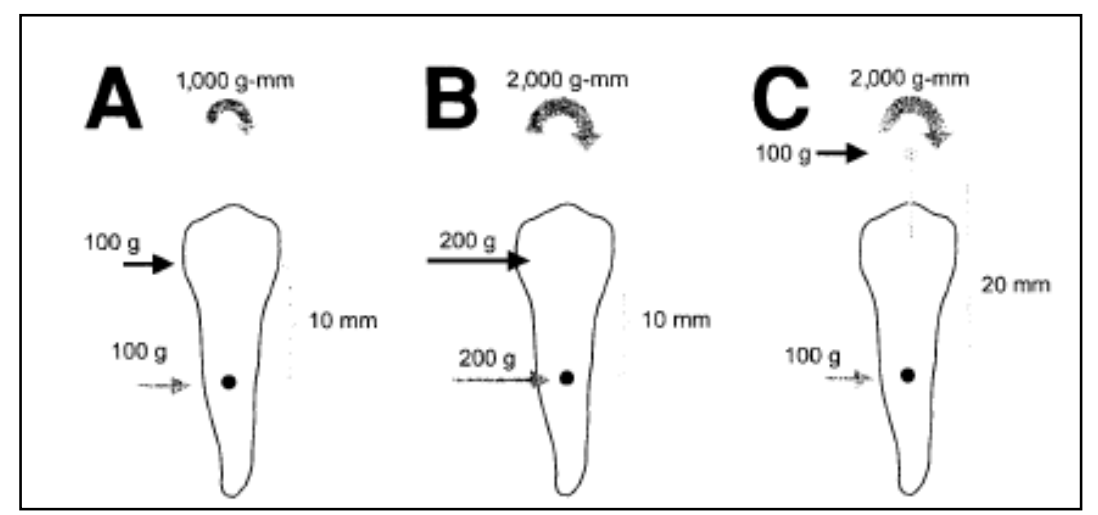

Fig. 8: The magnitude of a moment depends on both the magnitude of the applied force and its perpendicular distance from the center of resistance, $M=F d$; $(A)$. The magnitude of the moment increases as the force increases; $(B)$ or the distance increases $(C)$ 


\section{Moment to Force Ratio ${ }^{15}$}

At whatever point a force is connected at the crown of a tooth, an inclination for the tooth to rotate, tip, or torque (moment) is made. Notwithstanding the force connected, a couple may likewise be locked in deliberately to partially correct, totally right, or overcorrect this propensity. By changing the proportion existing moment from the connected couple to the force connected, the center of rotation of the tooth moment can be shifted to create the kind of tooth moment wanted. A clinical illustration is solitary, distally coordinated force acting at the bracket of a tooth on a consistent arch wire. This force results in a moment of force tending to move the center of resistance toward the force and furthermore to pivot the tooth around its. To accomplish in bodily movement we need to neutralize this tendency of the tooth to tip a clockwise way by drawing in a second order couple to turn the tooth in the contrary counter clockwise direction. An ideal harmony among the moment to force to turn the tooth a clockwise way and a tendency of moment of couple to pivot the tooth in a counter clockwise way would create tooth translation. In connected orthodontic wording, this relationship is communicated as a proportion of moment of couple to the force that creates the moment of power, or the moment of force proportion $(\mathrm{M} / \mathrm{F})$. For instance distal canine translation is desired and a force of $100 \mathrm{~g}$ is connected $10 \mathrm{~mm}$ coronal to the center of resistance. This will tend to move the center of resistance distally toward the force vector. Since the force isn't acting through the center of resistance, an inclination for distal crown tip or MF will likewise be available. The moment or inclination to pivot toward this path is quantitated at $100 \mathrm{~g} * 10 \mathrm{~mm}=$ $1000 \mathrm{gmm}$. At the point when the wire is seated in an edgewise bracket, an inclination to pivot the tooth the other way is given by a Mc made by a second order couple at the bracket. In the event that the bracket is $4 \mathrm{~mm}$ long, an arch wire would need to apply $250 \mathrm{~g}$ at each end of bracket to make the fundamental Mc of $1000 \mathrm{gmm}$ the other way. In the event that this could be accomplished, each of the inclinations for revolution would be disposed off and the net tooth movement would be translation toward the applied force. ${ }^{16}$

The easiest method to decide how a tooth will move is to consider the proportion between moment made when a force is connected to the crown of a tooth (the moment of a power, or MF) and the counter balancing moment created by a couple inside the bracket (the moment of the couple, or Mc).

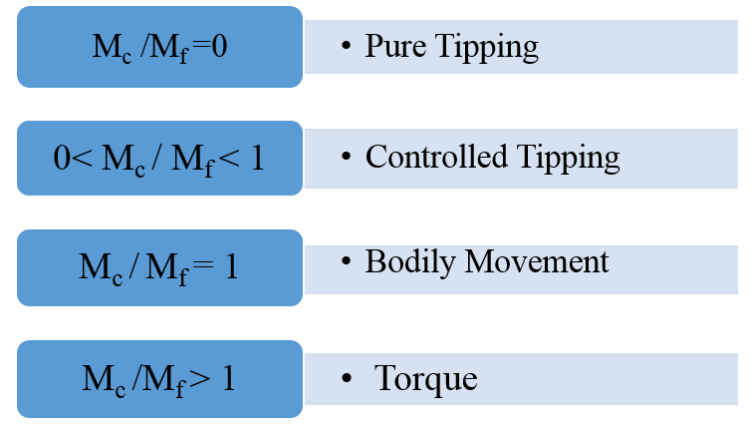

$\mathrm{M} / \mathrm{F}$ ratios are dealt along with individual tooth movements.

\section{Couple $^{17}$}

A couple includes two forces of comparable degree with parallel yet non collinear lines of action and opposite senses. ${ }^{11}$

The two forces of a couple check any tendency for the center of resistance of the tooth to move, anyway the minute made by the two forces don't drop each other. The tooth, along these lines, turns about its forces of resistance paying little regard to the point of utilization of the couple. If the two forces of the couple follow up on opposite sides of the center of resistance, their effect to make a moment is added substance. In case they are on a comparable side of the center of resistance, they are subtractive. Regardless, no net force is felt by the tooth, only a penchant to turn. Thusly a couple applies an unadulterated moment to a tooth. The span of moment made by a couple is reliant on both force size and distance. The moment of a couple is the total of the moments made by each of the two forces that make up a couple:

Moment of couple $=$ moment of force $1+$ moment of force 2 or

Moment of couple $=$ F1D1 + F2D2

$\mathrm{F} 1$ and F2 are equal but opposite. Therefore, magnitude of a moment created by a couple is F (D1 $+\mathrm{D} 2)$ or $\mathrm{F}$ times the distance between the two forces, $\mathrm{M}=\mathrm{FD}$.

A couple applies no net force on the center of resistance because the two forces that include it are reverse in bearing and drop each other. Thus, a couple alone reliably acts to turn, tip, or torque the tooth around the center of resistance. In addition, the extent of moment made by a couple depends just on the measure of its forces and the detachment between them, not on the partition of the couple from the center of resistance. In this way, the couple acts the same on a tooth paying little regard to the point where it is connected. A theoretically basic instance of a couple is a premolar that is turned by expanding an elastic one route from the buccal connection and another from the lingual connection the other way (Fig. 9). In this way two proportionate and inverse forces are associated and 
the tooth pivots its center of resistance without translation.

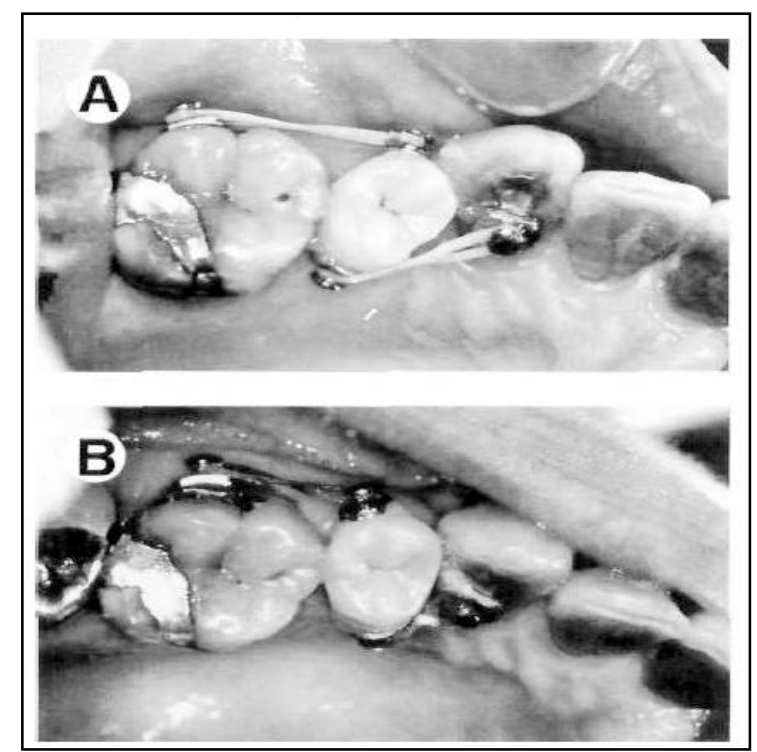

Fig. 9: A couple is applied to derotate a premolar. Before (A) and after (B)
Couples are associated by attracting a wire in an edgewise bracket slot. To achieve a first order couple, 18 the wire is angulated to make equal size of power at the mesial and distal parts of the bracket in inverse, buccal and lingual directions as showed up in the Fig. 10A. To make a second order to couple, the wire produces equal yet inverse intrusive and extrusive forces at the mesial and distal viewpoints as in Fig. 10B. A curve or torque in the wire will convey intusive and extrusive forces that are identical and inverse acting at the buccal and lingual parts of the bracket slot to make a third order couple as showed up in the Fig. 10C.

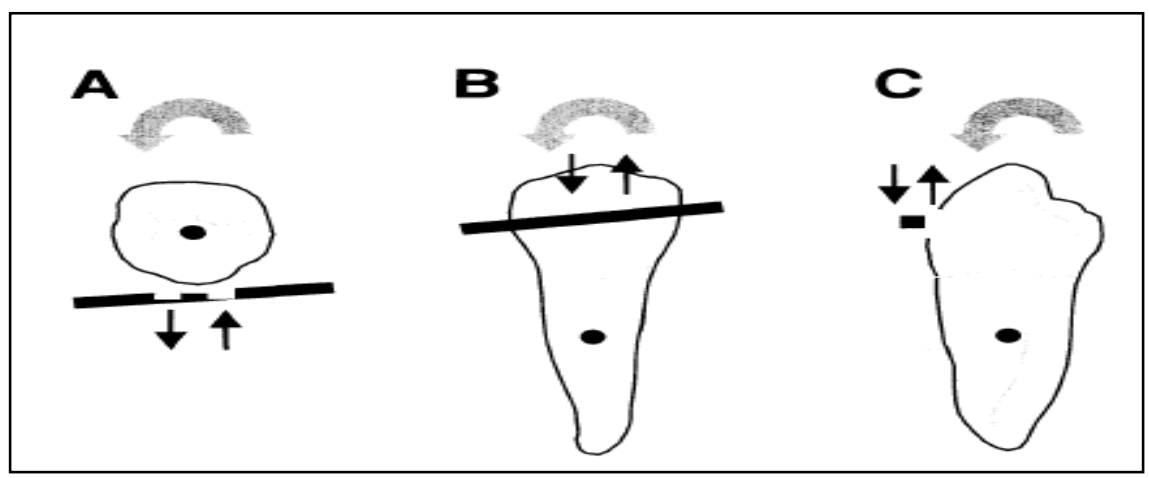

Fig. 10: Couple is frequently made in orthodontics by embeddings an active wire into an orthodontic bracket. The wire applies comparable and opposite noncollinear powers (dark) achieving an unadulterated moment (gray). Occlusal view (A), buccal view (B), mesial view (C)

\section{Static Equilibrium}

At the point when orthodontic appliances are activated and inserted, tooth movement does not happen promptly but rather continues gradually finished over an expanded timeframe. Consequently, the physical laws of statistics are viewed as satisfactory to depict the immediate force frameworks delivered by orthodontic appliances. The laws of static's can't be utilized to depict how the force framework will change as a tooth moves and an appliance de activates and modifies its arrangement. Newton's laws govern the physical laws of statistics. The three laws are as follows. ${ }^{19}$

1. The law of inertia: Everybody continues in its state of rest or uniform motion in a straight line unless it is compelled to change by the forces impressed on it.

2. The law of acceleration: The change in motion is proportional the motive force impressed and is made in the direction of straight line in which the force is impressed.

3. The law of action and reaction: For every action there is equal and opposite reaction.

The orthodontic application of these laws is for each appliance, however not really for each tooth to which it is appended, the aggregate of the forces and the entirety of moments must be equivalent to zero.9 That is, on account of the appliance itself does not move in a split second once it is placed, the net force framework delivered by the appliance all in all must be equivalent to zero. Each orthodontic appliance paying little mind to the treatment logic utilized must meet this condition, it is difficult to outline an appliance that characterizes the laws of physics. The analysis of equilibrium can be stated in the equation form

$\sum$ horizontal forces $=0$

$\sum$ Vertical forces $=0$ 
$\sum$ Transverse forces $=0$

And,

$\sum$ moments (Horizontal axis)

$\sum$ Moments (vertical axis)

$\sum$ Moments (Transverse axis)

a) Equal and opposite forces:

The straightforward orthodontic appliance break down is the elastic band. An elastic band extended between two points of connection will create a force of same magnitude an one end and, by the laws of equilibrium deliver a contrary force of a similar extent at the opposite end. To the extent the elastic band is concerned, it is in harmony. The total of the forces delivered by elastic, equivalent and inverse, is zero. ${ }^{20}$

One couple appliances - statically determinate systems.

A one couple orthodontic appliance is inserted into a bracket or tube toward one side and it tied as a point contact at the other. ${ }^{10}$ Since it isn't locked in into an orthodontic bracket, the end i.e tied as a point contact, can't create a couple however just a straightforward power at that site. The opposite end which is occupied with the bracket slot, can deliver both a force and couple at that connection. The appliance is a $1-$ couple system in light of the fact that a couple is produced just at the site of full engagement. It is statically determinate on the grounds that the magnitude of the forces and movements delivered can be resolved clinically after the appliance is embedded into the bracket. This should be possible by embeddings the appliance into the bracket and measure the force required to activate the wire to the site where it will be tight as a point contact. A case of a 1 - couple orthodontic appliance is long arm or cantilever activated to extude a high maxillary buccal canine (Fig. 12). The wire is embedded into the molar auxilary tube and bent to rest coronal to the canine. It is activated by displacing the wire apically and it to the canine bracket to make a extensive force (Fig. 11A). ${ }^{20}$

The most predictable approach to determine the force system delivered by any orthodontic appliance including a 1-Couple appliance is to remove the activated wire from its attachments and lay it latently over the attachment site. The angle formed by the wire and the bracket will demonstrate the direction of the couple created at site of engagement where the angle between the wire and bracket is largest. ${ }^{11,13}$ Because there is just a single site of engagement in a 1-Couple framework, the course of the couple at the site depends just on the angle formed between that bracket and the wire as appeared in (Fig. 11B). The molar bracket must turn counter clockwise to connect with the wire creating a couple to tip the molar crown mesially and root distally. The laws of equilibrium direct that the aggregate of the moments applied by the wire be equivalent to zero. With a counter clockwise moment at the molar, there must be a clockwise moment to maintain equilibrium. That moment is delivered by intrusive and extrusive forces of equivalent size applied by the wire at the molar and canine, respectively, as shown in (Fig. 11C).

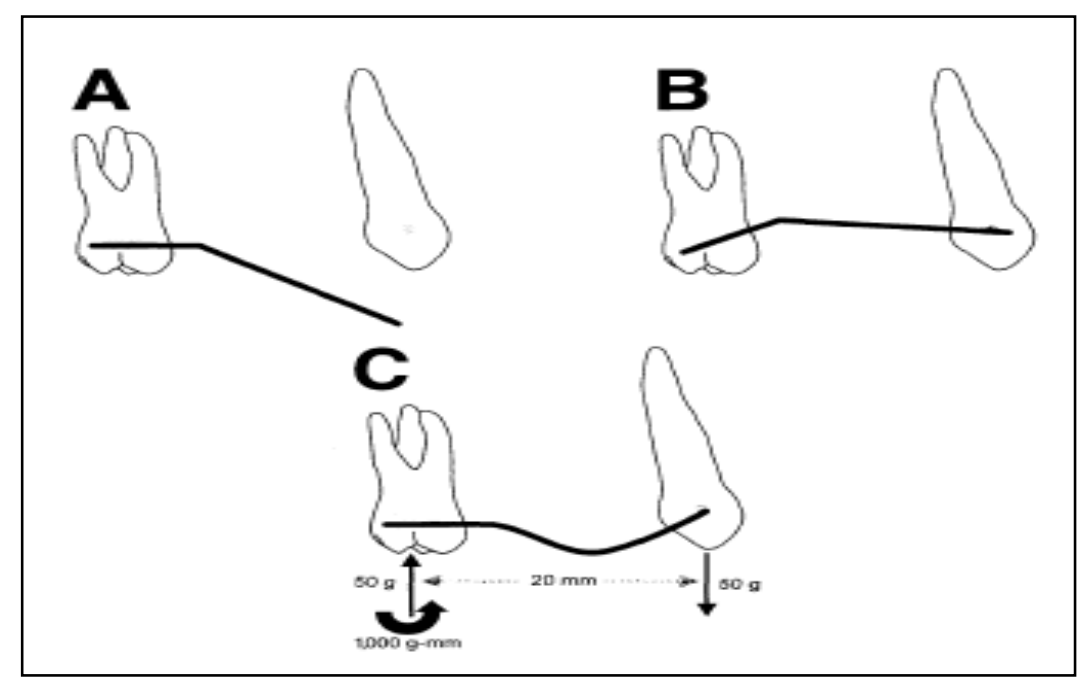

Fig. 11: A long arm or cantilever to extrude a high canine (A). The passive wire demonstrating the angle between the molar bracket and the wire $(B)$. The force system applied by the wire is in balance with a crownmesial momemt at the molar, and equivalent and inverse extrusive and intrusive forces at the canine and molar, individually (C). Moments, so the aggregate of the moments is additionally zero and the conditions of static harmony are met

Two - Couple appliance statically indeterminate

A 2 couple appliance is one that is locked in into systems. $^{21}$ attachments at the both ends. The wire at either or both connection locales creates a couple. For this situation, embeddings the wire into one attachments and utilizing 
a gauge to check the forces required for diverting the wire to the other bracket won't really give an exact appraisal of the force created by the appliance. In view of the powerlessness to quantify force system created by 2 - couple appliance. Clinically, they are alluded statistically vague. The force system delivered by 2 couple orthodontic appliance rely upon both the wire geometry and bracket angulations relationship. The powers that can be applied by a 2 - couple appliance might be found by latently putting the wire over both attachment sites to decide the angle formed between the bracket slot and the wire at each end. In the event that the angle formed at the two connections are equivalent and inverse, equivalent and inverse couples will be created and no force will be produced at either side, as appeared in (Fig. 13A). On the off chance that the forces are equivalent and in a similar direction, at that point the two sites will encounter couples a similar direction and of equivalent greatness. The force produced at the attachment sites will be equivalent in size and inverse in direction to each other. Together, the force will form a couple on the wire to keep up equilibrium of the appliance overall. The couple formed by the two forces will be inverse in course and equivalent in extent to the sum of two couples delivered at the individual connection sites as appeared in (Fig, 13B).

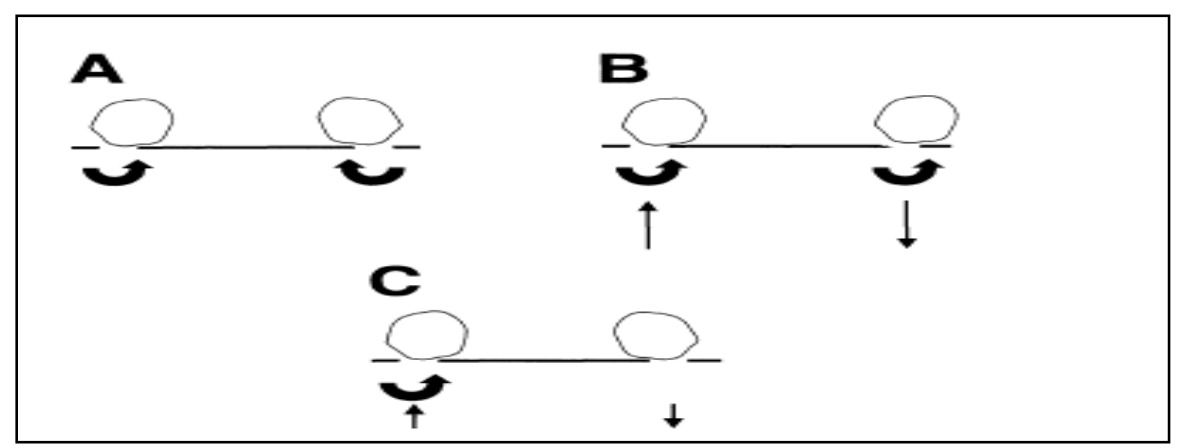

Fig. 13: Force systems from a 2-couple appliance rely upon wire-bracket geometry. Equivalent and inverse bracket-wire angle connections result in equivalent and inverse couples without any forces; (A). Equal bracket wire angle relationships in a similar direction result in equal couples in a similar direction with huge force to keep up appliance equilibrium; $(B)$. In the event that bracket-wire angle connections are unequal, the attachment with the biggest angle will have the biggest couple and resultant forces will be toward a path inverse this moment to look after harmony $(\mathrm{C})$.

In the event that the angle formed between the latently placed wire and two brackets slots are not equivalent in size, at that point the site with the greatest angle can be resolved this will be the site at which the greatest couple will be produced. The direction of the moment created at that tooth will be what will convey the bracket to the wire. The forces at the two attachment sites will be equivalent in magnitudes and inverse in direction to each others as usual. Together, these forces will frame a couple on the wire to keep up balance of the appliance as a whole. ${ }^{13,15}$ The couple shaped by the two forces will be inverse in bearing to the couple applied at the site of attachments with the greatest bracket - wire angulations as appeared in (Fig. 13C). The size of the forces and the direction of the couple at the other connection site, be that as it may, may not be evident from clinical perception alone. The relative moments and forces created by two - couple appliance activation are greatly delicate to clinical geometry. They have been determined for 2 dimensional and 3 -dimensional conditions. ${ }^{22}$

Burstone and Koenig in 1974. At the point when the two brackets are similarly however oppositely angled as appeared in (Fig. 14A), the resultant couples are mirror images of each other (their numerical total is zero) and no forces are produced. ${ }^{24}$ A non straight diminishment of the correct moment happens as the angulations of the right bracket is diminished marginally in Fig. as the supreme whole of the two moments increase on account of the adjustment in relative bracket angulation, corresponding increments in the forces are noticed that keep up the states of equilibrium (Fig. 14B). At the point when the angle of right bracket is a half of that of the left, yet at the same time inverse in direction, no couple is available at the right bracket, as represented in (Fig. 14C). Moment in the right bracket are in the same direction and left bracket couple increases as the right bracket angle declines to zero and after that increases to emulate the left section angulations (Fig. 14D). At long last, when both brackets are equivalent in direction and angulations, couples are additionally equivalent (their mathematical sum is maximal, and harmony forces are at maximum (Fig. 14E). Similarly, the static force systems produced by setting wires with capital $\mathrm{V}$ and step-bends into adjusted co planner brackets have been all around reported. ${ }^{14}$ In a two dimensional model using two brackets, a symmetrically placed V-Bend produced equal and oppositely directed. 


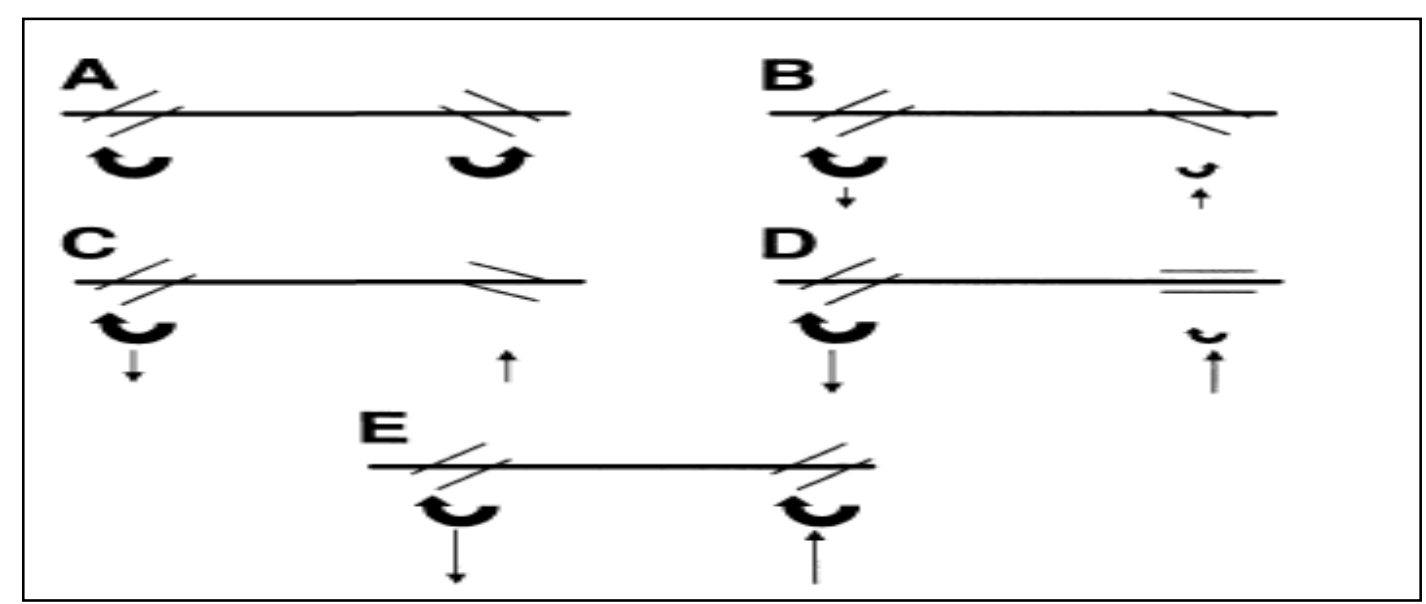

Fig. 14: Force systems from a 2-couple appliance: straight fragment of wire in malaligned brackets

Equal and oppositely calculated sections result in equivalent and inverse couple at that bracket angulation diminishes, the greatness of the couple at that bracket is a half of that of the forces result (B). At the point when the angulation of one bracket with the smaller angulation and forces are therefore expanded (C). As the bracket angle keeps on diminishing toward one side of the appliance, the couple at the lesser angled bracket is an indistinguishable way from at the greater angles bracket and forces are considerably more prominent (D). At the point when the two brackets are equivalent and a similar direction, forces are at maximum (E). At the point when the summit of the $\mathrm{V}$ bend is halfway between the couples, (Fig. 15A). A symmetric bend result in different combinations of moments and forces.
Moving the bend slightly off center results in an expansion in the couple produced at the bracket nearer to the bend and a diminishing at the bracket further away, an appeared in (Fig. 15B). A step bend, paying little heed to where it is put, results in bend is situated at $1 / 3$ of the separation between two brackets as a result of the deflection angle of the activated wire, a couple results at just the bracket closer to the bend (Fig. 15C). Forces increase to look after balance. Bends closer to the bracket result in couples at the two brackets in same direction with the more prominent movement created at the bracket closed to the band (Fig. 15, D), the two couples are now additive, and much more noteworthy forces result at the two connections to keep up harmony of the appliance. ${ }^{24}$

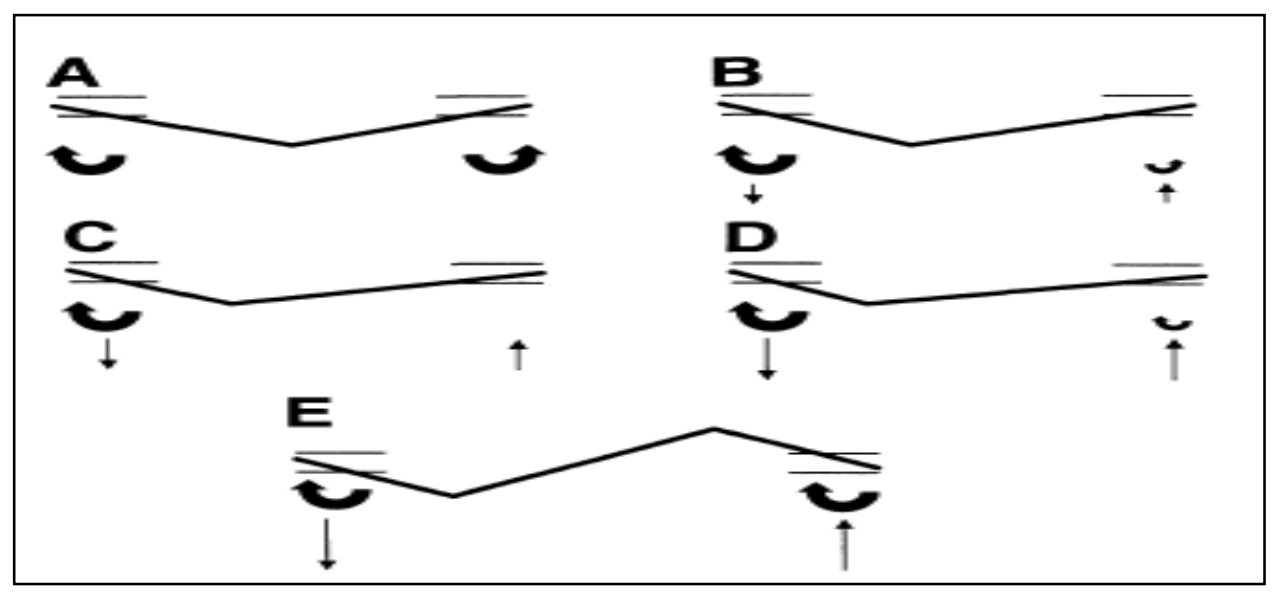

Fig. 15. Forces systems from a 2-couple machine: bowed wire in adjusted brackets.

Step bends are less sensitive than V-Bends to area and result in moments of equivalent size and direction at the two brackets free of where they are put as appeared in (Fig. 15E), with couples of equivalent size created at both attachment sites in a similar direction, forces are expanded further to keep up harmony conditions. The forces and moments appeared in figures 16 and 17 are correct for 2 - couple appliances dynamic in any one measurement. The brackets, along these lines, are appeared without teeth to accentuate that the force systems might act in the occlusal, lateral, or frontal planes. Relative sizes of the arrow and curves reflects contrast in force and moment size, respectively equal couples a similar direction at both attachment. ${ }^{25}$ 


\section{References}

1. Gandin LG Jr, Gandini MR, Amaral RM. Continuous torque system with control of the reaction unit. Am J Orthod Dentofacial Orthop. 2010;137:393-5.

2. Viecilli RF. Self-corrective T-loop design for differential space closure. Am J Orthod Dentofacial Orthop. 2006;129:48-53.

3. Choy K, Pae EK, Kim KH, Park YC, Burstone CJ. Controlled space closure with a statically determinate retraction system. Angle Orthod. 2002;72:191-8.

4. Kuhlberg AJ, Burstone CJ. T-loop position and anchorage control. Am J Orthod Dentofacial Orthop. 1997;112:12-8.

5. Shroff B, Yoon WM, Lindauer SJ, Burstone CJ. Simultaneous intrusion and retraction using a three-piece base arch. Angle Orthod. 1997;67:455-61.

6. Kalra V, Burstone CJ, Nanda R. More on fixed magnetic appliances. Am J Orthod Dentofacial Orthop. 1990;97:27A-28A.

7. Manhartsberger C, Morton JY, Burstone CJ. Space closure in adult patients using the segmented arch technique. Angle Orthod. 1989;59:205-10.

8. Burstone CJ. The segmented arch approach to space closure. Am J Orthod. 1982;82:361-78.

9. Melsen B, Fotis V, Burstone CJ. Vertical force considerations in differential space closure. J Clin Orthod. 1990;24:678-83.

10. Sifakakis I, Pandis N, Makou M, Eliades T, Bourauel C. Forces and moments on posterior teeth generated by incisor intrusion biomechanics. Orthod Craniofac Res. 2009;12:305-11.

11. Badawi HM, Toogood RW, Carey JP, Heo G, Major PW. Three-dimensional orthodontic force measurements. Am J Orthod Dentofacial Orthop. 2009;136:518-28.

12. Cattaneo PM, Dalstra M, Melsen B. Moment-to-force ratio, center of rotation, and force level: a finite element study predicting their interdependency for simulated orthodontic loading regimens. Am J Orthod Dentofacial Orthop. 2008;133:681-9.

13. Biomechanics of Tooth-Movement: Current Look at Orthodontic Fundamental 523.

14. Proffit WR, Fields HW Jr., Sarver DM. Contemporary Orthodontics. 2006, Elsevier Health Sciences, Edition 4.
15. Mulligan TF. Common sense mechanics in everyday orthodontics. 2009, CSM Publishing, Phoenix, Arizona, USA.

16. Burstone CJ, Pryputniewicz RJ. Holographic determination of centers of rotation produced by orthodontic forces. Am J Orthod. 1980;77:396-402.

17. Davidian EJ. Use of a computer model to study the force distribution on the root of the maxillary central incisor. Am J Orthod. 1971;59:581-588.

18. Hay GE. The equilibrium of a thin compressible membrane. Can J Res. 1939;17:106-121.

19. Yettram AL, Wright KWJ, Houston WJB. Center of rotation of a maxillary central incisor under orthodontic loading. Br J Orthod. 1977;4:23-27.

20. Christiansen RL, Burstone CJ. Centers of rotation within the periodontal space. Am J Orthod.1969;55:351-369.

21. Smith RJ, Burstone CJ. Mechanics of tooth movement. Am J Orthod.1984;85(4):294-307.

22. Pryputniewicz RJ, Burstone CJ. The effect of time and force magnitude on orthodontic tooth movement. J Dent Res. 58(8):1754-1764.

23. Mulligan TF. Common sense mechanics in everyday orthodontics. Phoenix, AZ: CSM Publishing; 1998:1-17.

24. Isaacson RJ, Lindauer SJ, Rubenstein LK. Activating a 2×4 appliance. Angle Orthod. 1993;63:17-24.

25. Demange C. Equilibrium situations in bend force systems. Am J Orthod Dentofacial Orthop. 1990;98:333339.

26. Koenig HA, Burstone CJ. Force systems from an ideal arch. Am J Orthod. 1974;65:270-289.

27. Burstone CJ, Koenig HA. Force systems from an ideal arch: large deflection considerations. Angle Orthod. 1989;59(1):11-16.

28. Koenig HA, Burstone CJ. Creative wire bending: the force system from step and V bend. Am J Orthod Dentofacial Orthop. 1988;93(1):59-67. 